\title{
Comfort-driven mobility produces spatial fragmentation in Axelrod's model
}

\author{
Sandro M. Reia, ${ }^{1}$ Paulo F. Gomes, ${ }^{1,2}$ and José F. Fontanari ${ }^{1}$ \\ ${ }^{1}$ Instituto de Física de São Carlos, Universidade de São Paulo, \\ Caixa Postal 369, 13560-970 São Carlos, São Paulo, Brazil \\ ${ }^{2}$ Instituto de Ciências Exatas e Tecnológicas, Universidade Federal de Goiás, 75801-615 Jataí, Goiás, Brazil
}

\begin{abstract}
Axelrod's model for the dissemination of culture combines two key ingredients of social dynamics: social influence, through which people become more similar when they interact, and homophily, which is the tendency of individuals to interact preferentially with similar others. In Axelrod's model, the agents are fixed to the nodes of a network and are allowed to interact with a predetermined set of peers only, resulting in the frustration of a large number of agents that end up culturally isolated. Here we modify this model by allowing the agents to move away from their cultural opposites and stay put when near their cultural likes. The comfort, i.e., the tendency of an agent to stay put in a neighborhood, is determined by the cultural similarity with its neighbors. The less the comfort, the higher the odds that the agents will move apart a fixed step size. We find that the comfort-driven mobility fragments severely the influence network for low initial cultural diversity, resulting in a network composed of only microscopic components in the thermodynamic limit. For high initial cultural diversity and intermediate values of the step size, we find that a macroscopic component coexists with the microscopic ones. The transition between these two fragmentation regimes changes from continuous to discontinuous as the step size increases. In addition, we find that for both very small and very large step sizes the influence network is severely fragmented.
\end{abstract}

\section{INTRODUCTION}

Homophily (i.e., the tendency of individuals to interact preferentially with similar others) and social influence (i.e., the tendency of individuals to become more similar with whom they interact) have long been perceived as major factors that influence social phenomena like segregation, inter-group bias and inequality, to mention only a few 1 3]. The understanding of the ways these factors impact social organization has been considerably expanded by the study of the agent-based model proposed by the political scientist Robert Axelrod in the late 1990s [4. Axelrod's model offered a simple quantitative approach to address the dissemination of culture among interacting agents in a society.

In Axelrod's model, the agents are represented by strings of cultural features of length $F$, where each feature can take on a certain number $q>1$ of distinct states (i.e., $q$ is the common number of states that each feature can assume). Hence the parameter $F$ represents the complexity of the society, since the different features are associated to different individual characteristics that are subject to social influence such as language, education, class, politics, religion, etc., whereas the number of states per feature $q$ represents the (potential) cultural diversity of the society - the larger $q$, the greater the number of options for the cultural features 4 .

The homophily factor is accounted for by the assumption that the interaction between two agents takes place with probability proportional to their cultural similarity (i.e., proportional to the number of states they have in common), whereas social influence enters Axelrod's model by forcing the agents to become more similar when they interact. Thus, there is a positive feedback loop between homophily and social influence: similarity leads to interaction, and interaction leads to still more similar- ity. Somewhat surprisingly, in spite of this homogenizing mechanism, Axelrod's model exhibits global polarization (i.e., a stable multicultural regime) for large $q$ in the case the agents are fixed to the sites of a square lattice and interact with their nearest neighbors only [4]. Variations of the original model have revealed that the relaxation of the homophilic interaction rules and the expansion of the interaction neighborhoods favor cultural homogenization (i.e., a stable monocultural regime) [5].

In the context of social organization, an important issue is the coevolution of the cultural states of the agents and the structure of the interaction or influence network (i.e., who interacts with whom) on the same time scale [10 15]. In fact, whereas in the original Axelrod model the agents are forced to interact with a predetermined group of agents (usually their nearest neighbors), we expect that in a more realistic scenario the agents would actively seek their likes and avoid their opposites. Such a scenario was considered for the nonlinear voter model 16, the weighted social network model [17] and the Sznajd model [18, where the network topology is allowed to change by (probabilistically) rewiring the links between agents with a bias towards the creation of links connecting agents with similar opinions or cultures.

In this paper we take a different approach and allow the agents to move in a square box by performing steps of fixed size $\delta$ in random directions in the plane. The agents are initially located at the nodes of random geometric graphs (RGGs) [19, where each node is randomly assigned geometric coordinates and two nodes are connected if the Euclidean distance between them is smaller than or equal to a certain threshold $d$. The set of agents connected to a particular agent defines its influence neighborhood. The RGGs were introduced in the 1960s to model wireless communication networks [19] and its connectivity properties were subsequently inves- 
tigated for threshold phenomena 20, 21. More recently, RGGs have been used to model synchronization [22], opinion dynamics 23, epidemic spreading 24] and epistemic communities 25 in scenarios where the agents are geographically constrained in certain regions.

The comfort-driven mobility is introduced in the model by assuming that the probability that a given agent stays put is proportional to the maximum value of its cultural similarity evaluated over all agents within its influence neighborhood. In addition, the agent moves with certainty if it is isolated, i.e., if its influence neighborhood is empty. Hence, an agent feels more comfortable (in the sense that it is less likely to move) in a location where at least one of its neighbors has a high homophily with it. These rules of motion are akin to those used in the modeling of the dynamics of human interactions with the cultural similarity playing the role of the social appeal between the individuals [26, 27]. We find that, for large systems, endowing the agents of Axelrod's model with the capacity to move in a plane following the comfort-driven rules of motion fragments the initially connected RGG into a macroscopic number of components and greatly suppress the culturally isolated agents, so most agents are comfortable in the absorbing configurations.

The rest of this paper is organized as follows. In section II we describe the rules of motion in the two-dimensional physical space where the agents roam freely as well as the rules that govern their interactions and determine how their cultural states change in time. In section III we present and analyze the results of our simulations, emphasizing the influence of the step size on the spatial and cultural organization of Axelrod's model with mobile agents. Finally, section IV is reserved to our concluding remarks.

\section{MODEL}

We consider a system of $N$ agents placed in a square box of linear size $L$ with periodic boundary conditions. In the initial configuration, the coordinates $x$ and $y$ of each agent are chosen randomly and uniformly over the length $L$. The density of agents $\rho=N / L^{2}$, which we fix to $\rho=1$ throughout this paper, yields the spatial scale for the interaction range $d$ and the step size $\delta$. In fact, since the effective area of an agent is $1 / \rho$, the quantity $d_{0}=1 / \sqrt{\rho}=1$ will be the standard to measure all distances in our study. More pointedly, we measure the distance $d$ within which interactions between agents are allowed in units of $d_{0}$, i.e., $d=d_{0} \alpha=\alpha$ with $\alpha>0$. The set of agents inside a circle of radius $d$ centered at a particular agent constitutes the influence neighborhood from where that agent selects a peer to interact with. Linking any two agents at a distance smaller than $d$ produces an undirected graph that we refer to as the influence network (see Fig. 1). Thus, as already pointed out, the initial disposition of the agents corresponds to the classic random geometric graph 19. We note that the

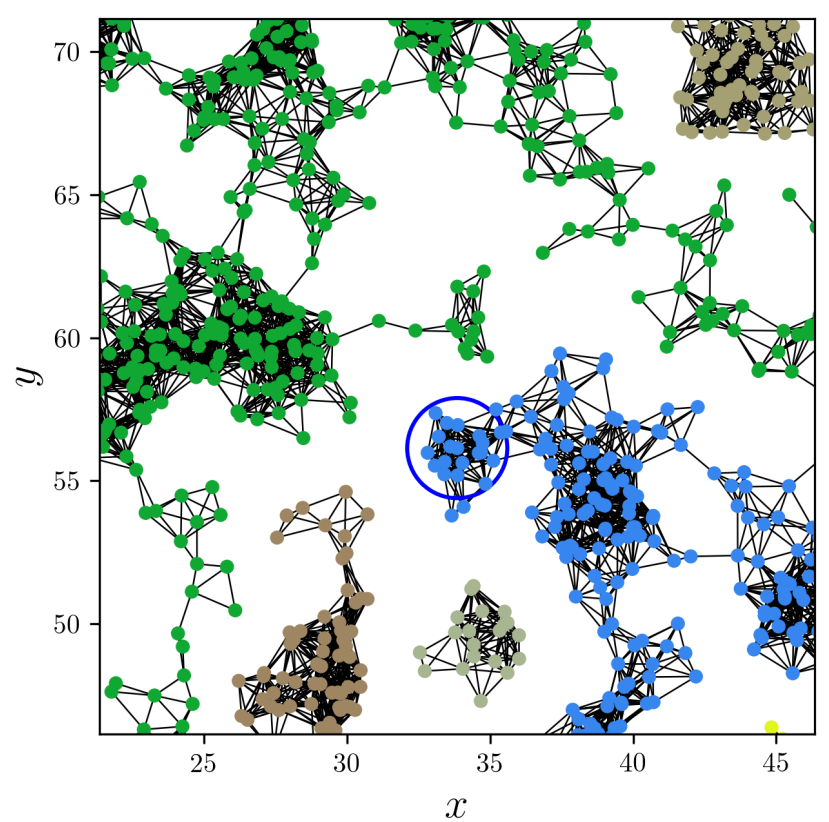

FIG. 1. Snapshot of a small portion of an absorbing configuration with $N=2^{15}$ agents and initial cultural diversity $q=10$. The display of the full configuration requires that the $\mathrm{x}$ and $\mathrm{y}$ axes range from 0 to $2^{15 / 2} \approx 181$. Agents within a distance $d=\alpha$, with $\alpha=1.75$, are connected by a link. The circle of radius $d$ centered at the central agent determines its influence neighborhood. The comfort-driven mobility with step size $\delta=9$ causes the fragmentation of the initial random geometric graph into a large number of components.

fixed value of the density $\rho$ is inconsequential, provided we use $d_{0}$ as the standard for measuring distances in the square box. However, the choice $\rho=1$ is consistent with the density of the regular square lattice, which was used in most studies of the Axelrod model [4].

In Axelrod's model, the initial states of the $F$ cultural features of the agents are drawn randomly from a uniform distribution on the integers $1,2, \ldots, q$. Once the initial configuration (i.e., the positions of the agents in the square box and their cultural features) is set, the dynamics proceeds as follows. It begins with the selection of an agent at random, the so-called target agent, and comprises two stages. The first stage is the motion on the square box and the second stage is the social interaction. To decide whether to move or not, the target agent evaluates its cultural similarity with all agents in its influence neighborhood and singles out the maximum value, which we denote by $a_{m} \in[0,1]$. (We recall that the cultural similarity between two agents is simply the fraction of features they have in common [4.) Here we assume that the target agent moves with probability $1-a_{m}$ and stays put with probability $a_{m}$. In the case the influence neighborhood of the target agent is empty, it moves with probability 1 . These rules of motion imply a sort of 'repulsion to the different' that prompts an agent to move away more frequently from neighborhoods that lack its 
cultural likes.

In the case the target agent decides to move, an angle $\theta \in[0,2 \pi)$ is chosen randomly to give the direction of motion and then a fixed step of length $\delta \geq 0$ is taken on that direction, similarly to the procedure used in the modeling of the dynamics of human interactions [27] as well as in the study of the effects of random motility on cooperative problem-solving systems 28 . Once the target agent is at the new position, a circle of radius $d=\alpha$ is drawn around it so that its (new) influence neighborhood is determined. Then the social interaction stage sets in: an agent within the influence neighborhood of the target agent is chosen at random and they interact with probability equal to their cultural similarity. An interaction consists of selecting at random one of the distinct features and making the selected feature of the target agent equal to the corresponding feature of its randomly chosen peer [4. In the case the target agent stays put, only the social interaction stage is implemented.

This procedure is repeated until the dynamics enters an absorbing configuration. According to the social interaction rule, absorbing configurations are such that agents within the influence neighborhood of a target agent are either identical to or completely different from it with respect to their cultural features. We note that the agents are not necessarily static in the absorbing configurations: in principle, an (non-interacting) agent that shares no cultural feature with any other agent in the system will keep moving forever without affecting or being affected by the established stationary social organization. However, we can easily identify this situation, which happens very rarely for $\delta>0$ since by construction the comfortdriven mobility aims at preventing the appearance of uncomfortable, i.e., culturally isolated, agents.

Once the dynamics reaches an absorbing configuration, we count the number of cultural domains $\left(\mathcal{N}_{d}\right)$ and record the size of the largest one $\left(\mathcal{S}_{d}\right)$, as usual [5] 9]. In time, a cultural domain is defined as a connected subgraph where the agents have the same culture (i.e., they share all cultural features). Hence, cultural diasporas are considered different cultural domains. In addition and more importantly, because of the rules of motion of the agents that, in principle, could allow them to organize themselves in isolated clusters or components, we measure also the number of components $\left(\mathcal{N}_{c}\right)$ and the size of the largest component $\left(\mathcal{S}_{c}\right)$ of the influence network. Since a component can sustain many cultural domains, we have $\mathcal{N}_{d} \geq \mathcal{N}_{c}$ and $\mathcal{S}_{c} \geq \mathcal{S}_{d}$. As all these quantities are bounded by the number of agents $N$, in section[II] we will characterize the absorbing configurations in terms of the densities $n_{d}=\mathcal{N}_{d} / N, s_{d}=\mathcal{S}_{d} / N, n_{c}=\mathcal{N}_{c} / N$ and $s_{c}=\mathcal{S}_{c} / N$.

Moreover, since our goal is to study the comfort-driven mobility, our study will focus mainly on the influence of the step size or mobility parameter $\delta$ on the statistical properties of the absorbing configurations. Accordingly, we will fix the parameter that determines the radius of the influence neighborhoods to $\alpha=1.75$. For large $N$ (and $\rho=1$ ) this choice produces initial configurations that are random geometric graphs with average degree per agent $\left\langle k_{i}\right\rangle \approx 9.62$, so the initial influence networks are almost surely connected graphs 20, 21. In addition, we will fix the number of cultural features to $F=3$ since this is the minimum value of $F$ for which the ordered and disordered regimes are stable in a large range of values of $q$ in the static case. Use of larger values of $F$ makes the convergence to the absorbing configurations prohibitively slow for large $N$. In the brief study of the static limit $\delta=0$, we will consider also the case $F=2$ in order to highlight the distinct nature of the phase transitions for $F=2$ and $F=3$.

For the sake of illustration, we show in Fig. 1 a snapshot of a portion of an absorbing configuration of a system of $N=2^{15}$ agents with $q=10$ and step size $\delta=9$. The influence neighborhood of the central agent is shown as a circle in the figure. The different components of this small portion of the influence network can be easily identified due to the absence of links between them. Next we will quantify the puzzling effects of the mobility parameter $\delta$ on the connectedness of the influence networks.

\section{RESULTS}

The measures we use to characterize the statistical properties of the absorbing configurations represent averages over (typically) $10^{3}$ independent runs, which differ initially by the cultural states of the agents as well as by their positions on the square box. As mentioned before, in the study of the effects of the mobility parameter $\delta>0$ we fix the radius of the influence neighborhood $\alpha$ and the cultural complexity $F$ of the system to $\alpha=1.75$ and $F=3$. The case $F=2$ is considered only for the static agents scenario, $\delta=0$.

\section{A. Static agents}

Because the Axelrod model was not studied in the case the agents are fixed at the sites of RGGs, it is instructive to consider briefly the static limit, $\delta=0$. Figure 2 shows the dependence of the fraction of agents in the largest cultural domain $\left\langle s_{d}\right\rangle$ on the initial diversity of the system $q$ for $F=2$ (upper panel) and $F=3$ (lower panel). This figure exhibits the hallmark of Axelrod's model for static agents, namely, the existence of a phase transition between ordered absorbing configurations, which are characterized by the presence of few cultural domains of macroscopic size (i.e., $\left\langle s_{d}\right\rangle$ is nonzero for $N \rightarrow \infty$ ), and disordered absorbing configurations, where all cultural domains are microscopic (i.e., $\left\langle s_{d}\right\rangle \rightarrow 0$ for $N \rightarrow \infty$ ). The phase transition is continuous for $F=2$ and discontinuous for $F=3$, similarly to the results for the square lattice [29, 30]. We note that the existence of the ordered phase implies a symmetry breaking leading to the dominance of few cultures, which does not happen 
for the one-dimensional model that, for $F=2$, exhibits disordered absorbing configurations for all $q$ [31, 32.

For the RGG, our results indicate that the continuous transition $(F=2)$ takes place between $q=6$ and $q=7$ and the discontinuous transition $(F=3)$ takes place between $q=19$ and $q=20$. We note that there is no need to use sophisticated methods (e.g., Binder cumulants 33) to determine the critical point $q_{c}$, since we can only state that $q^{*} \leq q_{c} \leq q^{*}+1$, where $q^{*}$ can be determined with almost certainty using the results of Fig. 2 , For instance, $q^{*}=6$ for $F=2$ and $q^{*}=19$ for $F=3$. In the static scenario, $q^{*}$ is the largest $q$ for which $\left\langle s_{d}\right\rangle>0$ in the thermodynamic limit. The order of the phase transition is determined by the presence or not of crossings of the curves of the order parameter $\left\langle s_{d}\right\rangle$ vs. $q$ for different system sizes.

Since the RGG is almost surely connected for $\alpha=1.75$, we have $\left\langle s_{c}\right\rangle \rightarrow 1$ and $\left\langle n_{c}\right\rangle \rightarrow 0$ for $N \rightarrow \infty$, regardless of the value of $q$. We recall that the topology of the influence network is not influenced by the social dynamics for $\delta=0$.

Figure 3 shows the mean fraction of culturally isolated agents $\left\langle\zeta_{d}\right\rangle$ for $F=2$ and $F=3$. Somewhat surprisingly, a large proportion of the agents are culturally isolated (i.e., they do not share any cultural feature with the agents in their influence neighborhoods) in the disordered regime of the static limit $\delta=0$. According to our definition, those agents are uncomfortable and would move with certainty if they were allowed to. We note that $\left\langle\zeta_{d}\right\rangle \rightarrow 0$ for $N \rightarrow \infty$ in the ordered regime. We advance that for $N$ and $q$ such that $N F / q \gg 1$, the comfort-driven mobility is very effective to suppress culturally isolated agents in the absorbing configurations (see A).

\section{B. Mobile agents for fixed system size}

Here we investigate the effects of the mobility parameter $\delta$ on the properties of the absorbing configurations for the system size $N=2^{16}$. Accordingly, Fig. 4 summarizes the influence of the initial diversity $q$ on $\left\langle s_{d}\right\rangle$ and $\left\langle s_{c}\right\rangle$ for several representative values of the step size $\delta$. The upper panel of the figure shows that the size of the largest cultural domain decreases with increasing $\delta$ and that the disruptive effect of the mobility parameter on $\left\langle s_{d}\right\rangle$ is enhanced for large $q$. These findings are consistent with the expectation that the comfort-driven mobility should increase cultural diversity (i.e., decrease $\left\langle s_{d}\right\rangle$ ) since it reduces the strength of social influence by decreasing the odds of repeated interactions between the same pair of agents. We advance, however, that our analysis of the finite size effects will show that $\left\langle s_{d}\right\rangle \rightarrow 0$ for all $q$ and $\delta>0$ in the thermodynamic limit, so the statistics of cultural domains is not informative in the context of mobile agents.

The low informative power of $\left\langle s_{d}\right\rangle$ contrasts with the mean size of the largest component $\left\langle s_{c}\right\rangle$, which is shown in the lower panel of Fig. 4 and highlights the strong influ-
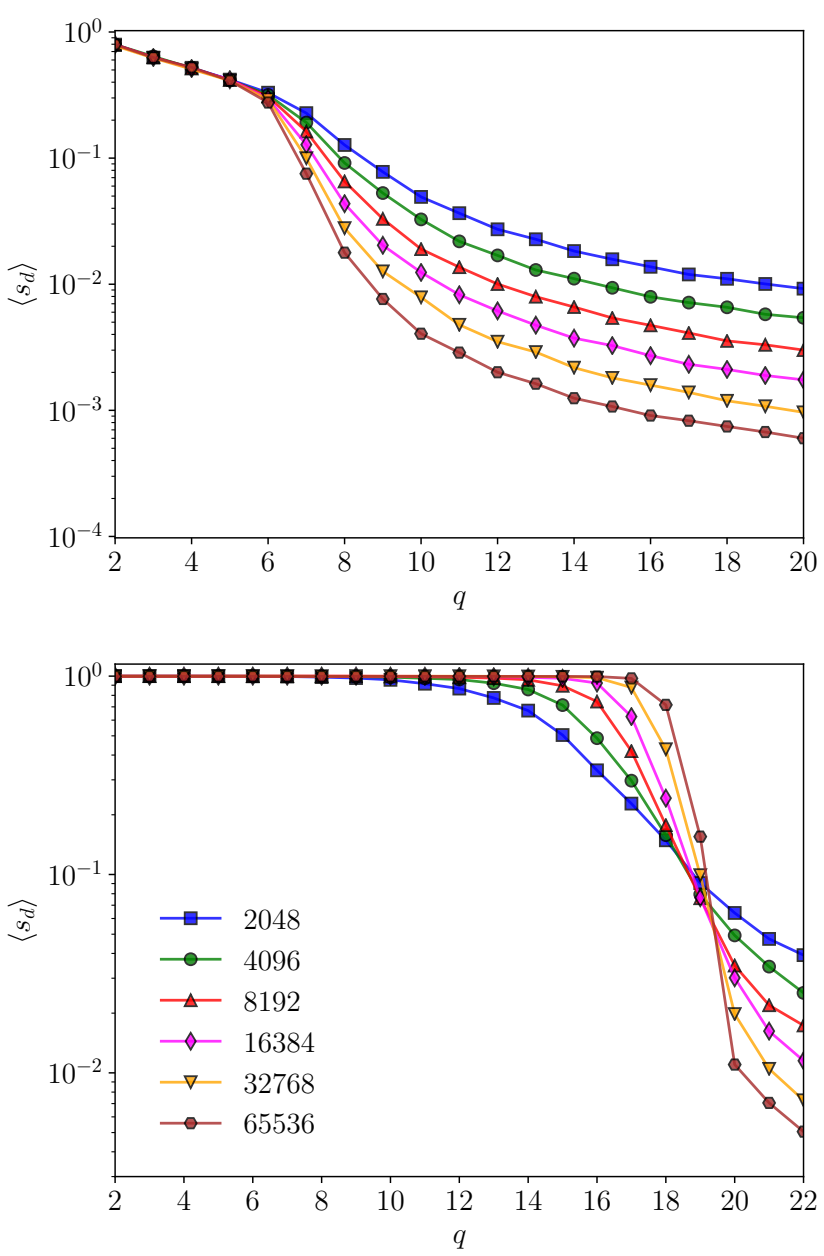

FIG. 2. Mean fraction of agents in the largest cultural domain $\left\langle s_{d}\right\rangle$ as function of the initial cultural diversity $q$ for $F=2$ (upper panel) and $F=3$ (lower panel). The agents are fixed at the sites of random geometric graphs (RGGs), i.e., the mobility parameter is $\delta=0$. The continuous phase transition for $F=2$ takes place between $q=6$ and $q=7$, whereas the discontinuous phase transition for $F=3$ takes place between $q=19$ and $q=20$. The system sizes are $N=2^{l}$ with $l=11, \ldots, 16$, as indicated.

ence of the model parameters on the connectedness of the influence network, manifested by the non-monotonous dependence of $\left\langle s_{c}\right\rangle$ on both $q$ and $\delta>0$. Recalling that $\left\langle s_{c}\right\rangle$ offers a picture of the fragmentation of the influence network, this panel shows that for small step sizes $\delta$ the fragmentation is more severe for low and intermediate values of the initial cultural diversity and that the network is almost connected for large $q$. For $\delta>6$, the influence network is severely fragmented regardless of the value of $q$. Interestingly, although the fragmentation of the influence network results necessarily in a decrease of the size of the largest cultural domain since $\left\langle s_{c}\right\rangle \geq\left\langle s_{d}\right\rangle$, the size of the largest cultural domain is actually smaller when the network is almost connected (i.e., $\left\langle s_{c}\right\rangle \approx 1$ ) than when it is severely fragmented. 

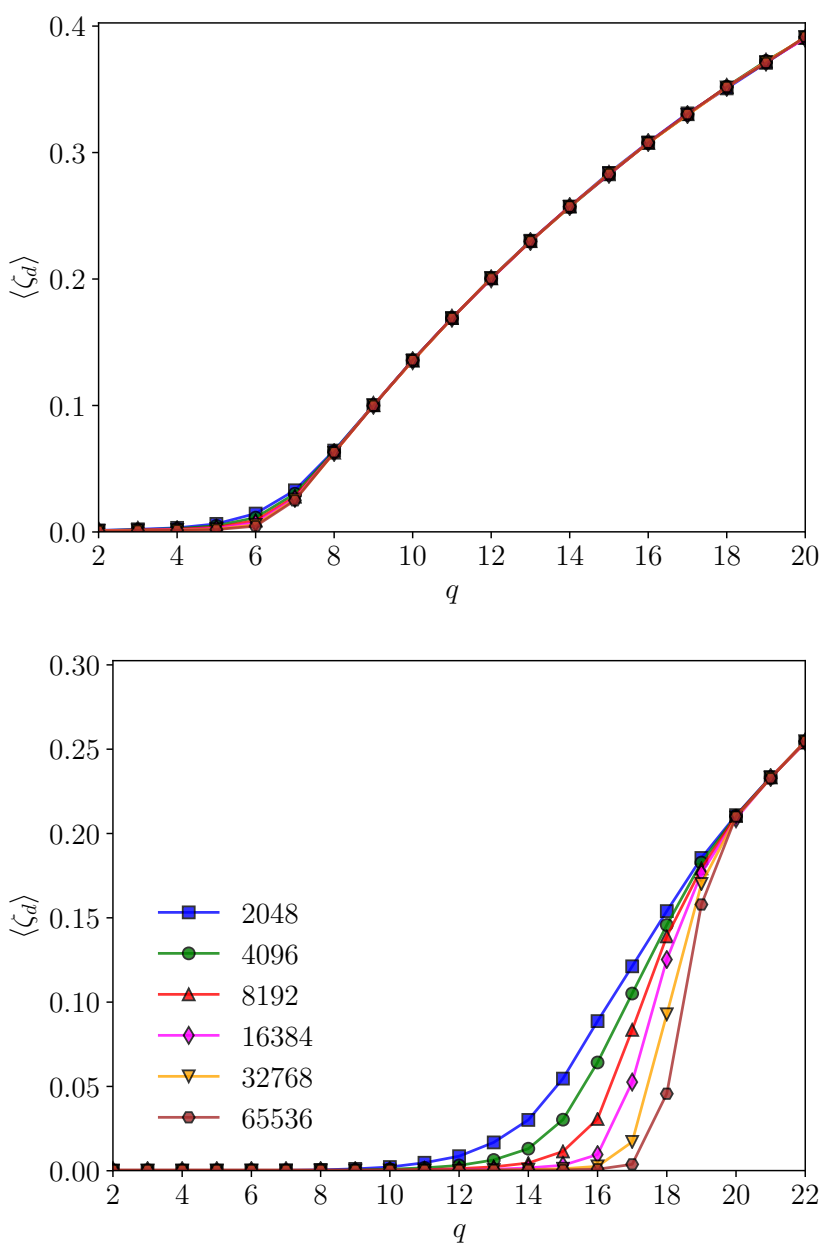

FIG. 3. Mean fraction of culturally isolated agents $\left\langle\zeta_{d}\right\rangle$ as function of the initial cultural diversity $q$ for $F=2$ (upper panel) and $F=3$ (lower panel). The agents are fixed at the sites of random geometric graphs (RGGs). i.e., $\delta=0$. The system sizes are $N=2^{l}$ with $l=11, \ldots, 16$ as indicated.

\section{Finite size effects}

Here we argue that the comfort-driven mobility induces a fragmentation transition separating the regime where all components are microscopic (i.e., $\left\langle s_{c}\right\rangle \rightarrow 0$ for $N \rightarrow \infty)$ from the regime where at least one component is macroscopic (i.e., $\left\langle s_{c}\right\rangle>0$ for $N \rightarrow \infty$ ). Henceforth we will refer to these regimes as the severely and mildly fragmented regimes.

Figures 5 and 6 summarize our results for $\delta=0.4$. In particular, the upper panel of Fig. 5 shows that $\left\langle s_{d}\right\rangle \rightarrow 0$ as $N$ increases, regardless of the value of $q$. More pointedly, for large $N$ we find that $\left\langle s_{d}\right\rangle$ vanishes as $N^{-0.8}$ for all $q$ (data not shown). The lower panel of this figure shows that the density of cultural domains $\left\langle n_{d}\right\rangle$ tends rapidly to its smooth asymptotic limit $\left\langle n_{d}\right\rangle_{\infty}>0$, indicating thus the presence of a macroscopic number of cultural domains for all $q$. Hence the comfort-driven mo-
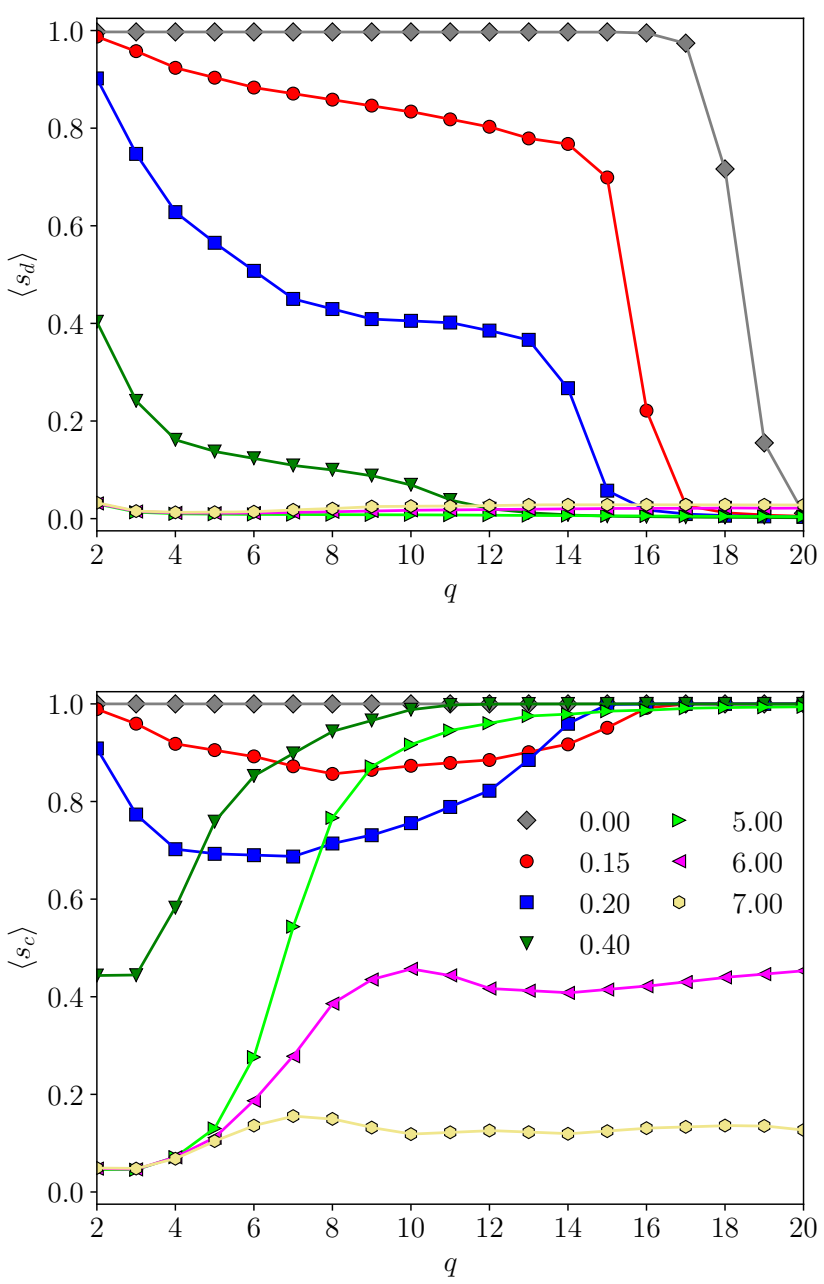

FIG. 4. Mean fraction of agents in the largest cultural domain $\left\langle s_{d}\right\rangle$ (upper panel) and in the largest component of the influence network $\left\langle s_{c}\right\rangle$ (lower panel) as functions of the initial cultural diversity $q$ for $N=2^{16}$ and step sizes $\delta=0,0.15,0.2,0.4,5,6$ and 7 , as indicated.

bility eliminates altogether the ordered absorbing configurations of the static limit. We recall that those configurations are the sole attractors of the dynamics in the range $q<20$ for $\delta=0$ (see lower panel of Fig. 2).

The left panel of Fig. 6 indicates that in the thermodynamic limit $\left\langle s_{c}\right\rangle$ exhibits a transition between $q=5$ and $q=6$ that separates the regime where the influence network is severely fragmented (i.e., all components are microscopic) from the regime where that network exhibits a macroscopic component. For instance, for $q=4$ we find that $\left\langle s_{c}\right\rangle$ vanishes like the power law $N^{-0.2}$ with increasing $N$, whereas it vanishes as $N^{-0.5}$ for $q=2$ (see Fig. 7).

We note that since $\left\langle s_{c}\right\rangle<1$ the influence network is always fragmented for $\delta>0$. The main difference between the severely and the mildly fragmented regimes is the presence or not of a macroscopic component in the thermodynamic limit. In addition, since the ratio $\left\langle s_{d}\right\rangle /\left\langle s_{c}\right\rangle$ 

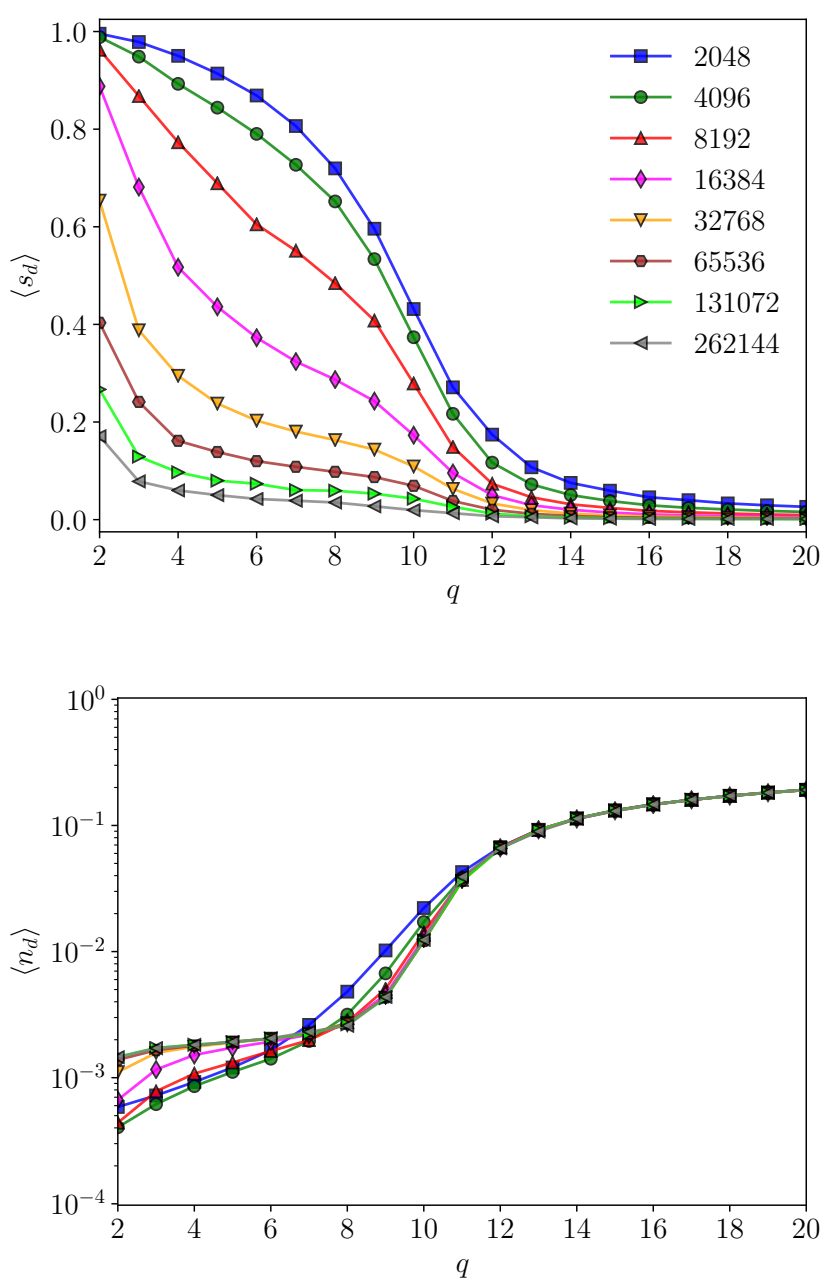

FIG. 5. Mean fraction of agents in the largest cultural domain $\left\langle s_{d}\right\rangle$ (upper panel), and mean density of cultural domains $\left\langle n_{d}\right\rangle$ (lower panel) as functions of the initial cultural diversity $q$ for $\delta=0.4$ and $N=2^{l}$ with $l=11, \ldots, 18$, as indicated.

tends to zero for large $N$ there is coexistence between different cultures inside the largest component in both fragmentation regimes.

The right panel of Fig. 6 shows that the mean density of components $\left\langle n_{c}\right\rangle$ is not affected by the fragmentation transition revealed in the study of the largest component. In addition, it shows that $\left\langle n_{c}\right\rangle>0$ in the limit $N \rightarrow \infty$, implying thus that the macroscopic component coexists with a macroscopic number of microscopic components for $q \geq 6$. This finding supports the claim that $\left\langle s_{c}\right\rangle\langle 1$ for $\delta>0$. From our results it is not possible to tell whether there are other macroscopic components in the mildly fragmented regime besides the largest one. Of course, since in the severely fragmented regime the largest component is microscopic (i.e., $\left\langle s_{c}\right\rangle \rightarrow 0$ for $N \rightarrow \infty)$, so are all the other components. The extrapolation of $\left\langle n_{c}\right\rangle>0$ to $N \rightarrow \infty$ (dashed curve in the right panel of Fig. 6) was obtained through the fitting
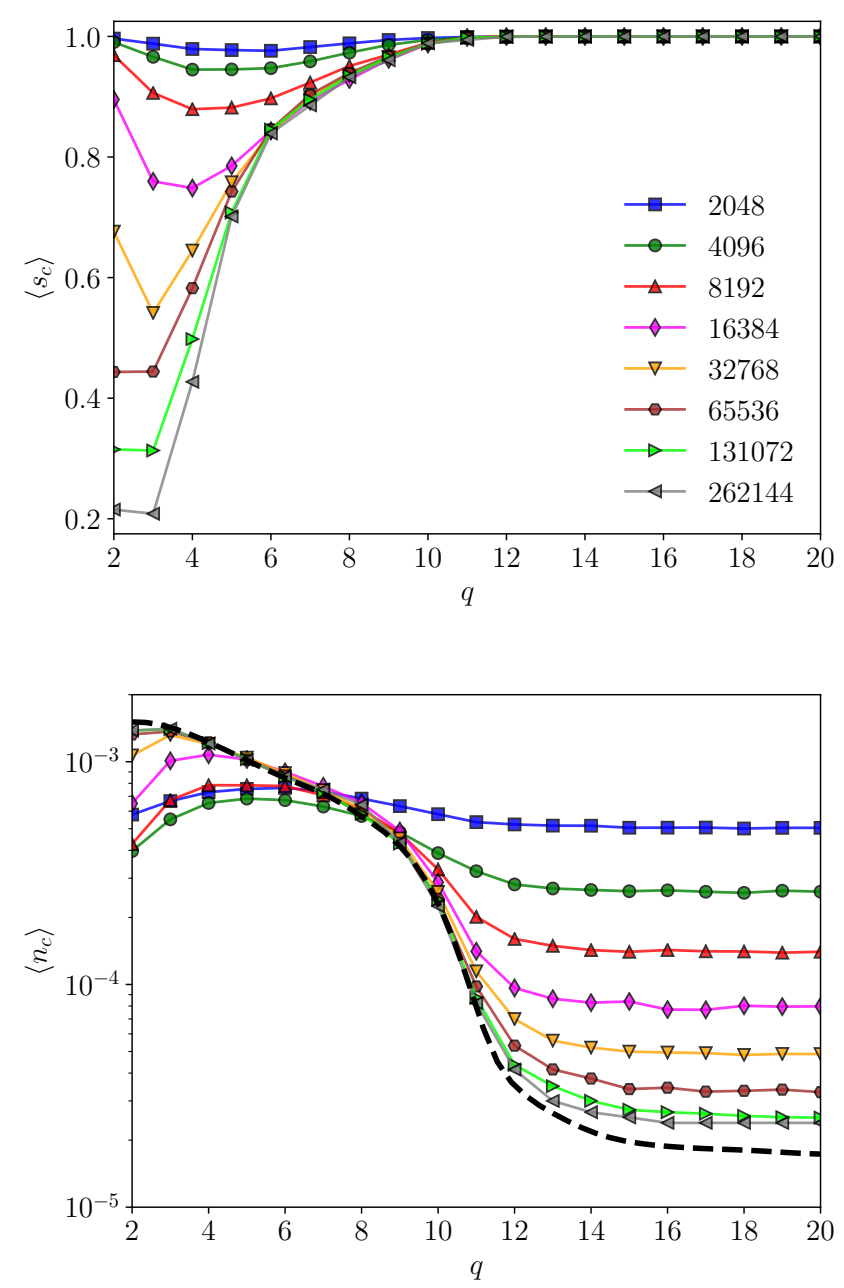

FIG. 6. Mean fraction of agents in the largest component of the influence network $\left\langle s_{c}\right\rangle$ (upper panel) and mean density of components $\left\langle n_{c}\right\rangle$ (lower panel) as functions of the initial cultural diversity $q$ for $\delta=0.4$ and $N=2^{l}$ with $l=11, \ldots, 18$, as indicated. The dashed curve in the lower panel is the fitting parameter $\left\langle n_{c}\right\rangle_{\infty}$ of the extrapolation of $\left\langle n_{c}\right\rangle$ to $N \rightarrow \infty$. The continuous transition separating the regime where the largest component of the influence network is macroscopic from the regime where all components are microscopic takes place between $q=5$ and $q=6$.

$\left\langle n_{c}\right\rangle=\left\langle n_{c}\right\rangle_{\infty}+a_{n} / N$ where $\left\langle n_{c}\right\rangle_{\infty}$ and $a_{n}$ are fitting parameters that depend on $q$ and $\delta$. Most interestingly, the parameter $a_{n}$ changes sign at about $q=6$, where the fragmentation transition takes place. Viewing $1 /\left\langle n_{c}\right\rangle$ as an estimator of the average component size, we can infer that when $a_{n}$ is positive $(q \geq 6)$, the components become bigger as the system size increases, which is consistent with the presence of a macroscopic component in the mildly fragmented regime. In turn, when $a_{n}$ is negative the components become smaller as $N$ increases, which is consistent with the existence of solely microscopic components in the severely fragmented regime.

The results for $\delta<0.4$ are similar to those exhibited 


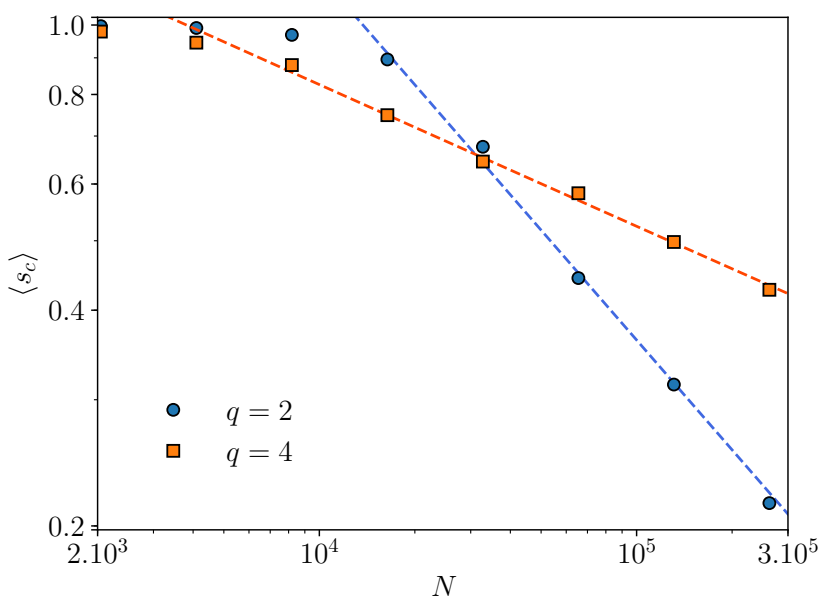

FIG. 7. Mean fraction of agents in the largest component $\left\langle s_{c}\right\rangle$ as function of the system size $N$ for the step size $\delta=0.4$ and $q=2,4$, as indicated. The dashed lines are the fittings $\left\langle s_{c}\right\rangle=135 N^{-0.51}$ for $q=2$ and $\left\langle s_{c}\right\rangle=5.24 N^{-0.20}$ for $q=4$.

in Figs. 5 and 6 but, as hinted at in Fig, 4 , the finite size effects are very strong, requiring the use of impracticably large system sizes to characterize the severely fragmented regime.

The normalized mean size of the largest component of the influence network $\left\langle s_{c}\right\rangle$ is the order parameter of the fragmentation transition and its dependence on the system size $N$ may shed some light on the nature of the phase transition. In particular, it would be of interest to know whether the transition between the severely and mildly fragmented regimes is continuous or discontinuous. Although it is somewhat problematic to discuss this classification in the case the independent variable $q$ is discrete, the fact that the curves of $\left\langle s_{c}\right\rangle$ vs. $q$ for different $N$ do not cross (left panel of Fig. 6) suggests that the fragmentation transition is continuous for $\delta=0.4$. It is interesting that the curves of $\left\langle n_{c}\right\rangle$ for distinct system sizes do cross at about $q=6$ (right panel of Fig. 6) but since $\left\langle n_{c}\right\rangle$ is not an order parameter (it is nonzero in both fragmentation regimes), it offers no information on the nature of the transition. These findings contrast starkly with the results for $\delta=5$ shown in Fig. 8, where the crossing of the curves for different system sizes happens for $\left\langle s_{c}\right\rangle$ but not for $\left\langle n_{c}\right\rangle$. This is the typical scenario of a discontinuous transition where the order parameter becomes independent of the system size at the threshold parameter $q_{c}$, which is thus determined by the intersection of the curves of $\left\langle s_{c}\right\rangle$ for large $N$. Since the relevant asymptotic behaviors, namely, $\left\langle n_{c}\right\rangle>0$ in the mildly fragmented regime and $\left\langle s_{c}\right\rangle \rightarrow 0$ in the severely fragmented regime, are more easily observed in Fig. 8 than in Fig. 6. we have not simulated the system sizes $N=2^{17}$ and $N=2^{18}$ for $\delta=5$.

We note that the nature of the fragmentation transition is determined by the dependence of $\left\langle s_{c}\right\rangle$ on $N$ in the
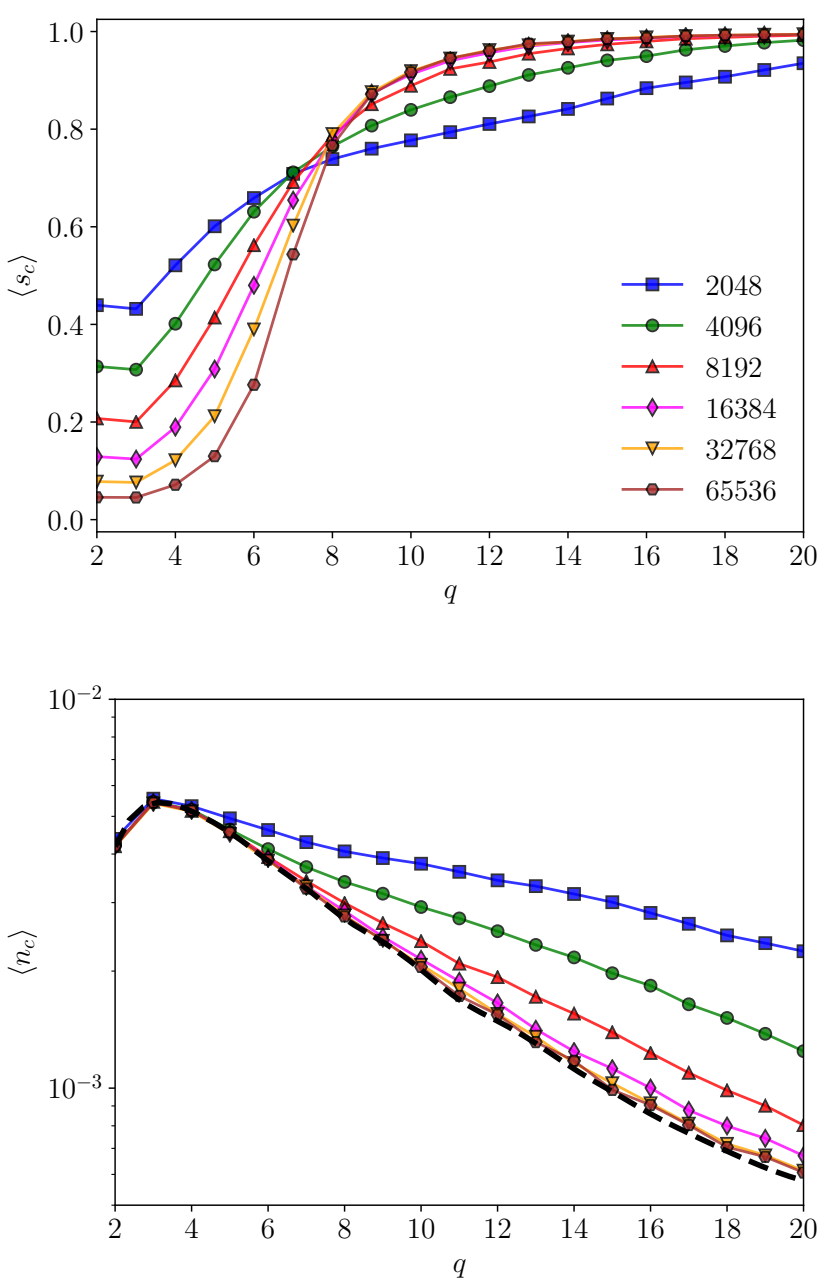

FIG. 8. Mean fraction of agents in the largest component $\left\langle s_{c}\right\rangle$ (upper panel) and mean density of components $\left\langle n_{c}\right\rangle$ (lower panel) as functions of the initial cultural diversity $q$ for $\delta=5$ and systems of size $N=2^{l}$ with $l=11, \ldots, 16$, as indicated. The dashed curve in the lower panel is the fitting parameter $\left\langle n_{c}\right\rangle_{\infty}$ of the extrapolation of $\left\langle n_{c}\right\rangle$ to $N \rightarrow \infty$. The discontinuous transition separating the regime where the largest component of the influence network is macroscopic from the regime where all components are microscopic takes place between $q=7$ and $q=8$.

mildly fragmented regime. For instance, in that regime $\left\langle s_{c}\right\rangle$ decreases with increasing $N$ for $\delta=0.4$ (see upper panel of Fig. 6), whereas it increases with increasing $N$ for $\delta=5$ (see upper panel of Fig. 8). In fact, in the mildly fragmented regime we can write $\left\langle s_{c}\right\rangle=\left\langle s_{c}\right\rangle_{\infty}+a_{s} / N$, where $\left\langle s_{c}\right\rangle_{\infty}$ and $a_{s}$ are fitting parameters, so we can use the sign of the parameter $a_{s}$ to determine whether the transition is continuous or discontinuous. Accordingly, we find that $a_{s}$ changes sign at $\delta \approx 4.2$, signaling thus a change on the nature of the fragmentation transition.

Now we consider an alternative method to estimate the transition points $q_{c}$, which relies on the analysis of the asymptotic behavior of the distribution of the com- 
ponent sizes 29. In particular, in Fig. 9 we show the cumulated distribution $U(S, q)$, which gives the fraction of components of size larger than $S \in[1, N]$. In the limits of large $N$ and $S$ such that $S \ll N$, we expect that $U\left(S, q<q_{c}\right) \rightarrow 0$ and $U\left(S, q>q_{c}\right) \rightarrow$ cte $>0$, so we can identify $q_{c}$ simply by observing the asymptotic behavior of this cumulated distribution. This approach yields the same estimates as those based on the order parameter $\left\langle s_{s}\right\rangle$, viz., that for $\delta=0.4$ the transition takes place between $q=5$ and $q=6$ (i.e., $q^{*}=5$ ) and that for $\delta=5$ it occurs between $q=7$ and $q=8$ (i.e., $q^{*}=7$ ). More importantly, however, is the fact that $U\left(S, q_{c}\right)$ decays as a power law $S^{1-\tau}$ and that the value of the exponent $\tau$ yields information on the nature of the transition at $q_{c}$ [29. More pointedly, the transition is continuous if $\tau \leq 2$ and discontinuous otherwise. The difficulty here is that because $q$ is discrete we cannot determine $q_{c}$ in
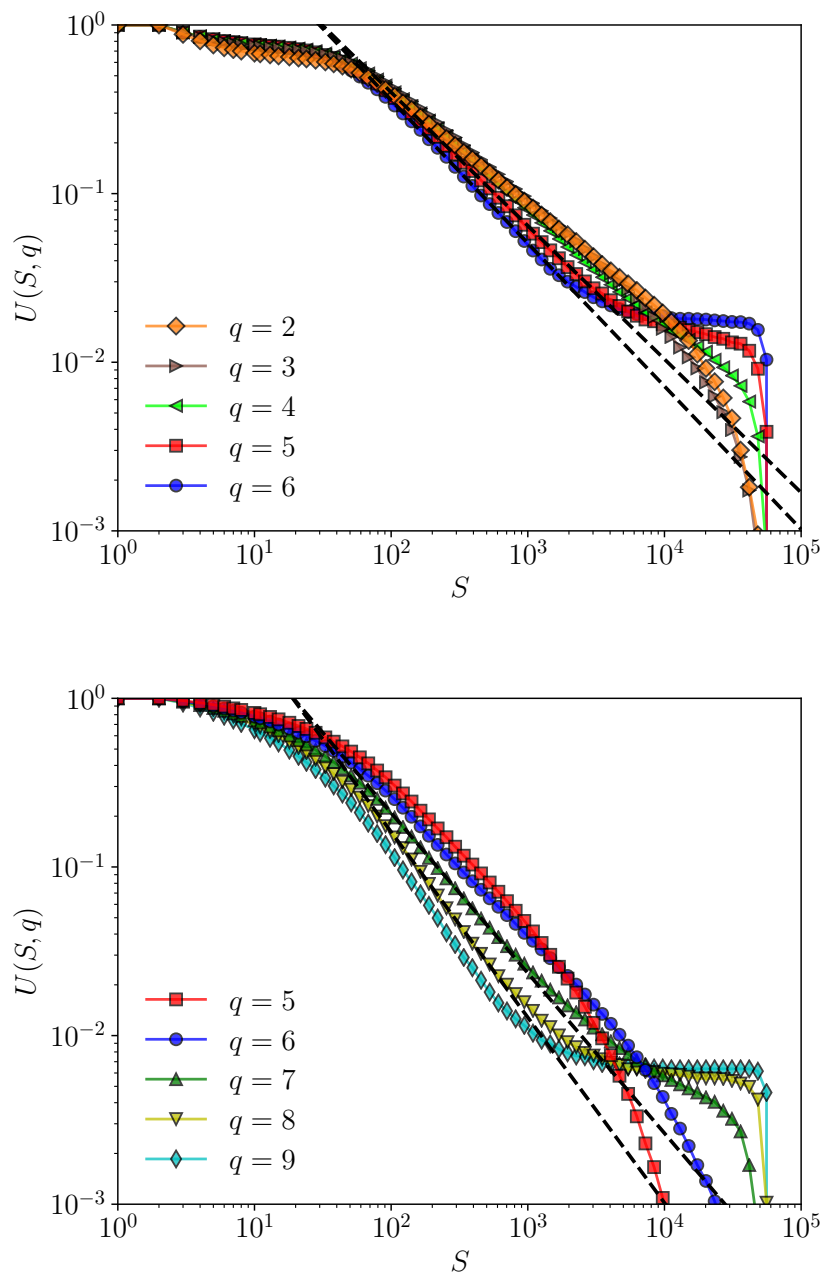

FIG. 9. Fraction of components of size larger than $S$ for $\delta=0.4$ (upper panel) and $\delta=5$ (lower panel). The system size is $N=2^{16}$ and the initial cultural diversities $q$ are as indicated. The dashed lines are the power-law fittings for $q=q^{*}$ and $q=q^{*}+1$ used to estimate the critical exponent $\tau$. order to observe the power decay. Nevertheless, in the regions where $U(S, q)$ at $q=q^{*}$ and $q=q^{*}+1$ can be approximate by power laws we find $\tau \approx 1.82$ for $\delta=0.4$ and $\tau \approx 2.05$ for $\delta=5$, which is consistent with the conclusions based on the crossing, not crossing of the curves showing system size effects. These estimates of the exponent $\tau$ are obtained by averaging the values of the slopes of the dashed straight lines for $q=q^{*}$ and $q=q^{*}+1$ shown in Fig. 9 .

As hinted at in Fig. 4, for $\delta=6$ and 7, the absorbing configurations remain highly fragmented even for large $q$. In fact, Fig. 10 summarizes the effects of the system size for these step sizes and shows that the absorbing configurations are severely fragmented in the thermodynamic limit, regardless of the value of $q$. This means that the transition between the severely and mildly fragmented regimes disappears altogether at a point $\delta^{*}$, so that for $\delta>\delta^{*}$ we have $\left\langle s_{c}\right\rangle \rightarrow 0$ for $N \rightarrow \infty$ for all $q$. The weak dependence on the system size observed for $\delta=6$ suggests that $\delta^{*}$ is close to 6 . In fact, we can obtain a rough estimate of $\delta^{*}$ by considering the dependence of $\left\langle s_{c}\right\rangle$ on $\delta$ for different system sizes, as shown in Fig. 11 for $q=10$. The critical value $\delta_{c} \approx 5.9$ was estimated by the intersection of the curves that fit the data of $N=2^{15}$ and $N=2^{16}$. In addition, we find that $\left\langle s_{c}\right\rangle \approx 0.5$ at $\delta=\delta_{c}$. A similar analysis for $q=20$ and $q=30$ yielded the same estimate for $\delta_{c}$ so we conjecture that $\delta^{*} \approx 6$.

The result that $\left\langle s_{c}\right\rangle \rightarrow 0$ for $\delta>\delta^{*}$ regardless of the value of $q$ holds true provided that $q$ is kept fixed while the system size $N$ approaches the thermodynamic limit. However, in the case $N$ is fixed and $q$ becomes arbitrarily large then the agents are likely to be moving all the time (without interacting as the probability of finding an agent with a common feature is very low) and the topology of the influence network becomes a sequence of RGGs. This scenario is discussed in A

Actually, the effect of the step size $\delta$ on the connectedness of the influence network is way more complicated than that suggested in Fig. 11. For instance, the results of Fig. 4 have already showed that for $q \in[4,14]$ and $N=2^{16}$ the network is more fragmented for $\delta=0.2$ than for $\delta=0.4$, which contrasts with the monotonous decrease of $\left\langle s_{c}\right\rangle$ with increasing $\delta$ exhibited in Fig. 11. This situation is seen more clearly in Fig. 12 that exhibits the region of small $\delta$ for different system sizes and reveals the existence of a valley in the curve $\left\langle s_{c}\right\rangle$ vs. $\delta$ that becomes deeper and closer to $\delta=0$ as $N$ increases. (We recall that $\left\langle s_{c}\right\rangle=1$ for $\delta=0$ in the thermodynamic limit.) The fragmentation observed for small $\delta$ is reminiscent of the fragmentation observed for small $q$ in Fig. 5 and, as in that case, we can only offer a conjecture about the asymptotic behavior of $\left\langle s_{c}\right\rangle$. Accordingly, we speculate that $\left\langle s_{c}\right\rangle$ vanishes continuously at $\delta_{c}$ for some $\delta_{c}>0$ so that in the thermodynamic limit $\left\langle s_{c}\right\rangle$ jumps from 1 to 0 as $\delta$ departs from 0 . We find it hopeless to estimate the continuous transition point $\delta_{c}$, which may be arbitrarily close to 0 , because $\left\langle s_{c}\right\rangle$ decreases very slowly with $N$ (see Fig. 12), thus making the simulation of the model near 

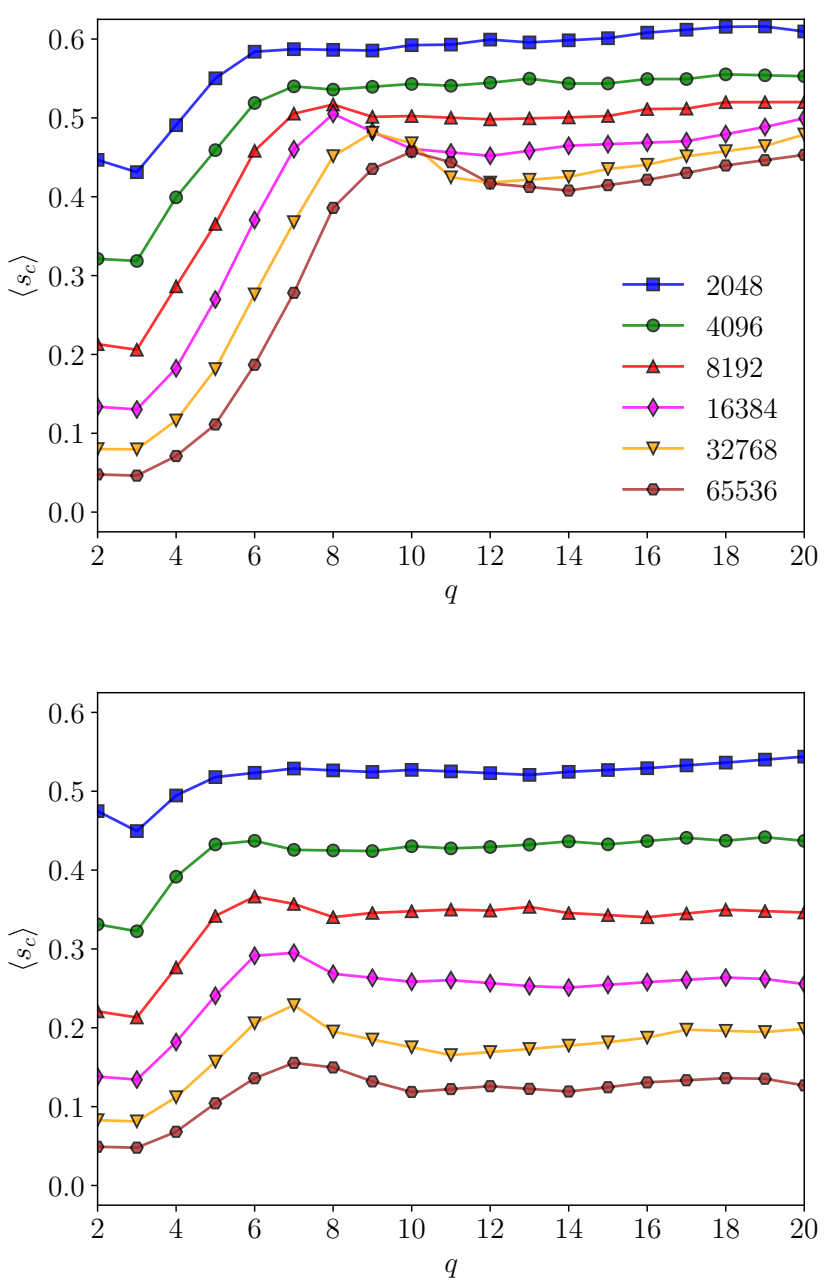

FIG. 10. Mean fraction of agents in the largest component $\left\langle s_{c}\right\rangle$ as function of the initial cultural diversity $q$ for $\delta=6$ (upper panel), $\delta=7$ (lower panel) and systems of size $N=2^{l}$ with $l=11, \ldots, 16$, as indicated.

the continuous transition point computationally impracticable.

In order to gain an insight into the nature of the mildly fragmented regimes for small and large step sizes, we present in Fig. 13 snapshots of two absorbing configurations for $\delta=0.2$ and $\delta=5.6$, which exhibit largest components with approximately the same size (viz., $s_{c} \approx 0.8$ ). It is evident that what distinguishes these configurations is the presence of other large components for small $\delta$, which could also be inferred by the fact that, other things being equal, there are many more components in the configuration for $\delta=5.6$ than in the configuration for $\delta=0.2$.

Before concluding, a word is in order about the microscopic mechanism that leads to the severe fragmentation regime for small $\delta$. This unexpected phenomenon can be understood with aid of the pair correlation function $g_{\delta}(r)$ shown in Fig. 17 of $\mathrm{B}$ and of the absorbing config-

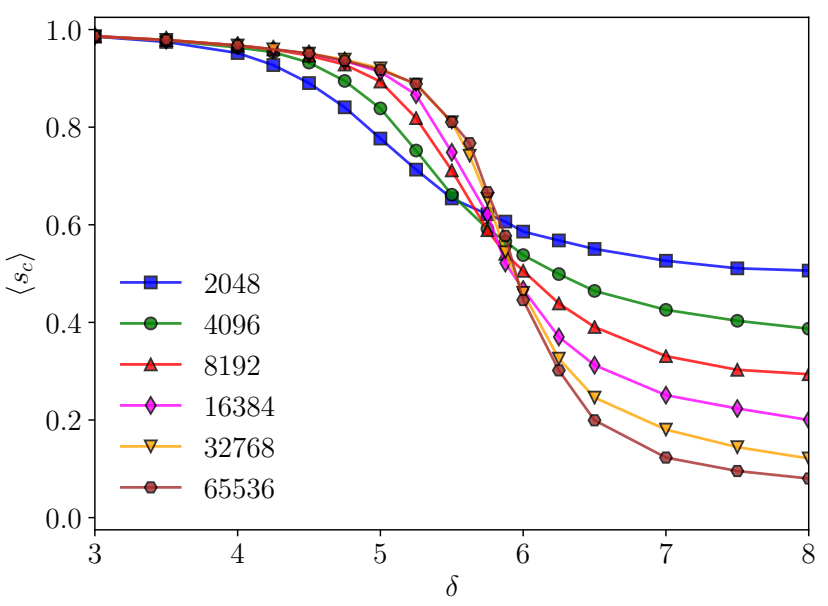

FIG. 11. Mean fraction of agents in the largest component $\left\langle s_{c}\right\rangle$ as function of the step size $\delta$ for $q=10$ and systems of size $N=2^{l}$ with $l=11, \ldots, 16$, as indicated. The discontinuous transition separating the regime where the largest component of the influence network is macroscopic from the regime where all components are microscopic takes place at $\delta \approx 5.9$.

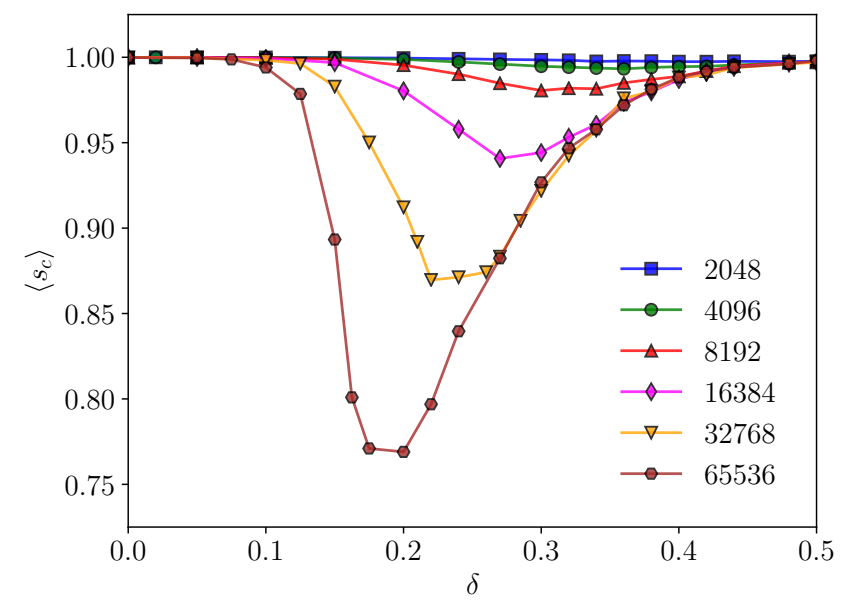

FIG. 12. Mean fraction of agents in the largest component $\left\langle s_{c}\right\rangle$ as function of the step size $\delta$ for $q=10$ and systems of size $N=2^{l}$ with $l=11, \ldots, 16$, as indicated.

uration shown in the upper panel of Fig. 13 for $\delta=0.2$. The small step size enhances the effect of social influence since $\delta \ll \alpha$ guarantees that many interactions between a same pair of agents will happen before one of them has the chance to leave the influence neighborhood of the other, which results in a relatively high density of pairs separated by distances smaller than $r=\alpha$. However, since for small step sizes the movement is practically continuous, the agents fine tune their motions to create exclusion regions where they would be uncomfortable, producing a dip in the pair correlation function for $r \in[2 \alpha, 3 \alpha]$ which explains the extraordinary similarity between the areas and shapes of the empty and occupied 

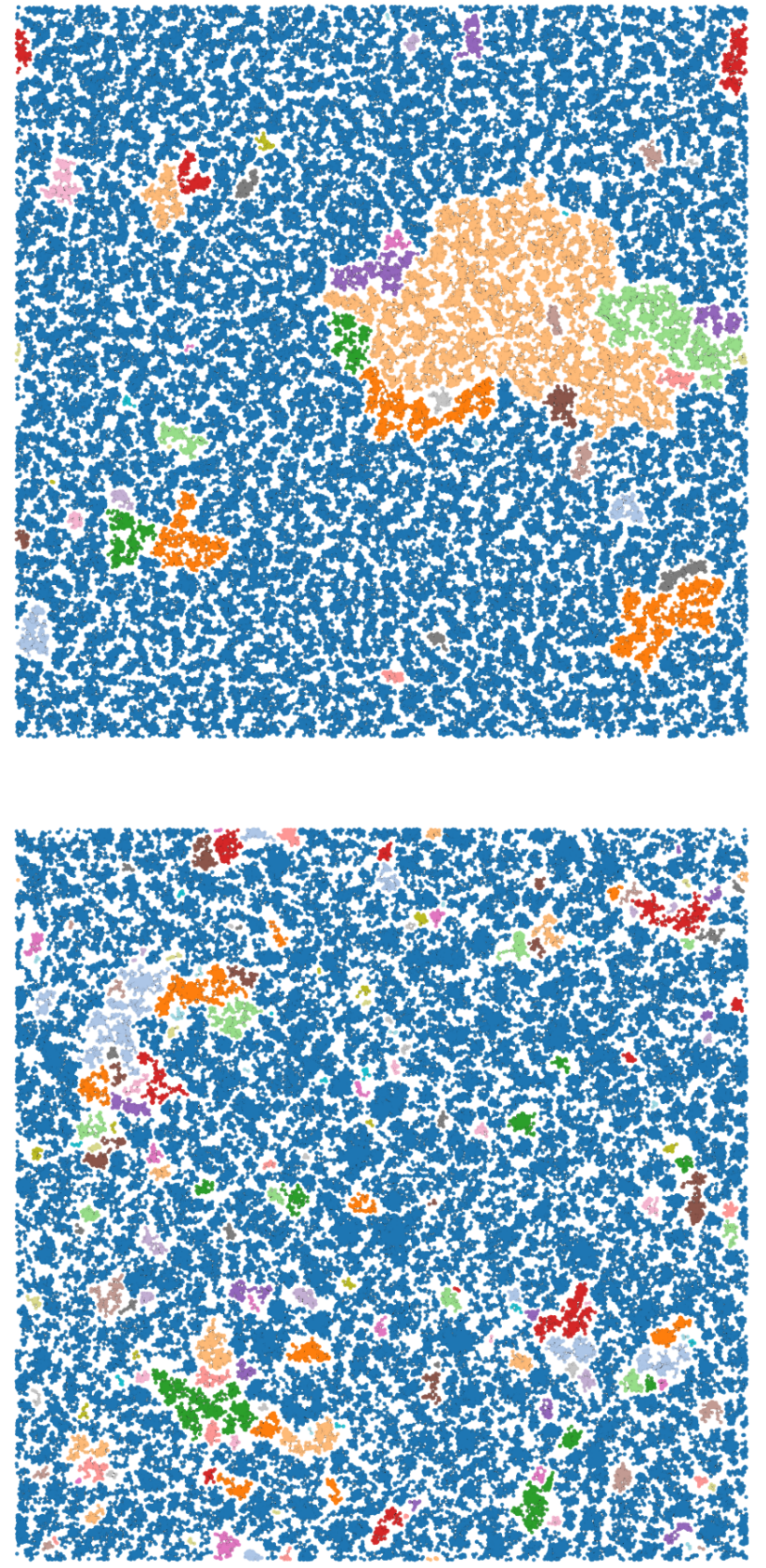

FIG. 13. Snapshots of two absorbing configurations showing the components of the influence network for $N=2^{16}$, initial cultural diversity $q=10$, step sizes $\delta=0.2$ (upper panel) and $\delta=5.6$ (lower panel). For $\delta=0.2$ we have $s_{c}=0.77$ and $n_{c}=0.0007$, whereas for $\delta=5.6, s_{c}=0.78$ and $n_{c}=0.003$.

regions in the upper panel of Fig. 13 Of course, exclusion regions with linear sizes greater than $\alpha$ lead to the fragmentation of the influence network.

Finally, to summarize our main results we offer in Fig. 14 a schematic portrayal of the phase diagram in the space $(\delta, q)$ showing the continuous and the discontinuous transition lines. We recall that for $\delta>\delta^{*} \approx 6$ the
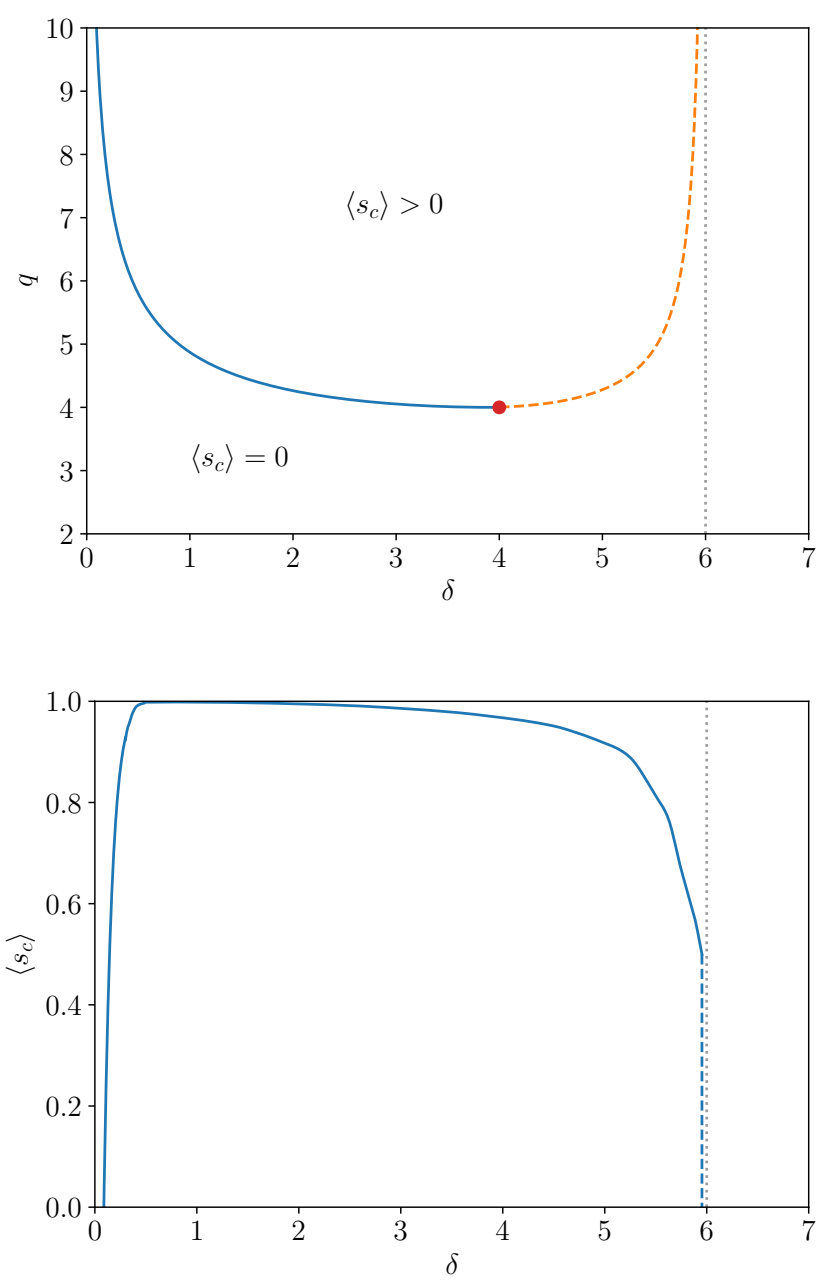

FIG. 14. Schematic phase diagram (upper panel) showing the continuous (solid curve) and discontinuous (dashed curve) transition lines that separate the severely $\left(\left\langle s_{c}\right\rangle=0\right)$ and mildly $\left(\left\langle s_{c}\right\rangle>0\right)$ fragmented regimes. The lower panel shows a schematic representation of the order parameter in the thermodynamic limit for $q=10$.

absorbing configurations are severely fragmented regardless of the value of $q$. This figure shows also a schematic depiction of the dependence of the order parameter $\left\langle s_{c}\right\rangle$ on $\delta$ in the thermodynamic limit, which was produced by the extrapolations of the finite size results exhibited in Figs. 11 and 12 for $q=10$.

\section{DISCUSSION}

Axelrod's model exhibits cultural diversity, in spite of the homogenizing effect of social influence, thanks to the rule that excludes the interactions between individuals that differ from each other in all their cultural features [4. Since in the original formulation of the model the agents are fixed at the nodes of a network, this rule amounts to the existence of a substantial number of 
uncomfortable (i.e., culturally isolated) agents that are stock-still away from their likes (see Fig. 3). A natural way to mitigate this frustration is to allow the agents to move away from their cultural antagonists. Here we allow the agents to move a fixed distance $\delta$ (step size) in random directions in the plane with a probability that depends on the maximum value of the cultural similarity evaluated over the agents in their influence neighborhoods. In particular, the greater that cultural similarity, the more comfortable the agent is and so the lesser the probability that it moves. We recall that in this paper we define the influence neighborhood of an agent as the region limited by a circle of radius $\alpha=1.75$ centered at the agent, and that the density of agents in the square box of linear size $L$ is set to $\rho=1$.

We hasten to note that an agent does not purposely move towards or away other agents in our model. However, the rule of motion which prescribes that the agent will stay put if it has an identical neighbor and that it will jump a distance $\delta$ in any direction if all its neighbors are antagonists results in an effective evasive behavior towards cultural antagonists, specially if $\delta>2 \alpha$. The explicit spatial scenario we consider here allows a clearer visualization and interpretation of the self-organized network components (see Fig. 13 and Fig. 17) as compared with approaches based on the rewiring of links [16, 17. In addition, our model offers a necessary development of the static scenario introduced in the original formulation of Axelrod's model since spatial proximity and mobility are key elements of the celebrated propinquity effect of social psychology, which is the tendency for people to form social bounds with those whom they encounter often 34. The drawback of our approach is that the simulation times to reach the absorbing configurations are much longer than for the original model, mainly due to the additional rules of motion and the need to keep a record of the positions of the agents in order to determine their influence neighborhoods. These add-ons limited the maximum system size we could simulate in a feasible time to $N=2^{18}$, although it is clear from Fig. 12 that much larger system sizes are necessary to bypass the strong finite size effects observed for small $\delta$.

We find that the introduction of the comfort-driven mobility in Axelrod's model produces a variety of startling results. In particular, we find that the influence network is fragmented for low initial cultural diversity $q$. We argue that this fragmentation is extreme, in the sense that all components of the influence network are of microscopic size in the thermodynamic limit (see Fig. 6). As $q$ increases, the system transitions to a mildly fragmented regime, which is characterized by the presence of a macroscopic component (i.e., $\left\langle s_{c}\right\rangle>0$ for $N \rightarrow \infty$ ). The value of the step size $\delta$ determines whether the transition between the regimes of severe and mild fragmentation is continuous or discontinuous: the fragmentation transition is continuous for $\delta<4.2$ and discontinuous otherwise. The discontinuous transition between the two fragmentation regimes disappears altogether for $\delta>6$, so the absorbing configurations are severely fragmented for all values of the initial diversity $q$. This severe fragmentation is not surprising for large $\delta$, since in this case an agent can quickly survey the entire plane to find its cultural likes and then freeze close to them. However, we find that the severe fragmentation occurs for small $\delta$ as well (see Fig. 4), thus resulting in a discontinuous jump of the relative size of the largest component at $\delta=0$ since the influence network is connected in the static limit. Regardless of the fragmentation regime, we find that the relative size of the largest cultural domain $\left\langle s_{d}\right\rangle$ vanishes in the thermodynamic limit, so the absorbing configurations are always multicultural.

The feedback between mobility and cultural similarity is responsible for these nonintuitive results, which make the behavior of Axelrod's model with comfort-driven mobility nonobvious logical consequences of the interaction rules, attesting thus the value and the need of the simulation model 35. In fact, the agent-based model proposed by Axelrod in the late 1990s has endured the test of time so far, probably because it exhibits the right balance between simplicity and realism as well as very intriguing critical phenomena 29 31. In this vein, introduction of comfort-driven mobility in Axelrod's model produced results of interest for the social sciences, such as the prevention of the formation of large cultural domains and the spatial segregation of the agents, as well as for statistical physics, such as the continuous and discontinuous fragmentation transitions of the influence network. Thus our results reaffirm Axelrod's model as a paradigm for idealized models of collective behavior [36].

\section{ACKNOWLEDGMENTS}

The research of JFF was supported in part by Grant No. 2017/23288-0, Fundação de Amparo à Pesquisa do Estado de São Paulo (FAPESP) and by Grant No. 305058/2017-7, Conselho Nacional de Desenvolvimento Científico e Tecnológico $(\mathrm{CNPq})$. SMR was supported by grant 15/17277-0, Fundação de Amparo à Pesquisa do Estado de São Paulo (FAPESP). Research carried out using the computational resources of the Center for Mathematical Sciences Applied to Industry (CeMEAI) funded by FAPESP (grant 2013/07375-0).

\section{Appendix A: Limit of high $q$}

For very high $q$ and fixed $N$, we expect that the absorbing configurations will not fragment for $\delta>0$ because the agents are likely to wander around the square box without interacting, since the probability of finding an agent with a common feature is very low for $q \gg 1$. The topology of the influence network becomes then a sequence of random geometric graphs (RGGs). To check 
this presumption we present in Fig. 15 the mean fraction of agents in the largest component $\left\langle s_{c}\right\rangle$ as well as the fraction of non-interacting (i.e., culturally isolated) agents $\left\langle\zeta_{d}\right\rangle$ for much larger values of $q$ than those used in the main text and for relatively small system sizes $N$. We recall that, for the step size $\delta=7$ used to generate these results, the influence network is severely fragmented in the thermodynamic limit. It is clear from our results that, for fixed $N$, an increase of $q$ will defragment the influence network according to our expectation. However, as $N$ increases, the defragmentation occurs for larger and larger values of $q$, which agrees with our conclusion that for any finite $q$ the influence network is severely fragmented in the thermodynamic limit.

The lower panel of Fig. 15, which exhibits the fraction of non-interacting agents, confirms that for the range of $q$ and $N$ considered in the main text, those agents are very rarely observed in the absorbing configurations produced by the comfort-driven mobility. In fact, even for the small system sizes show in this figure, the presence
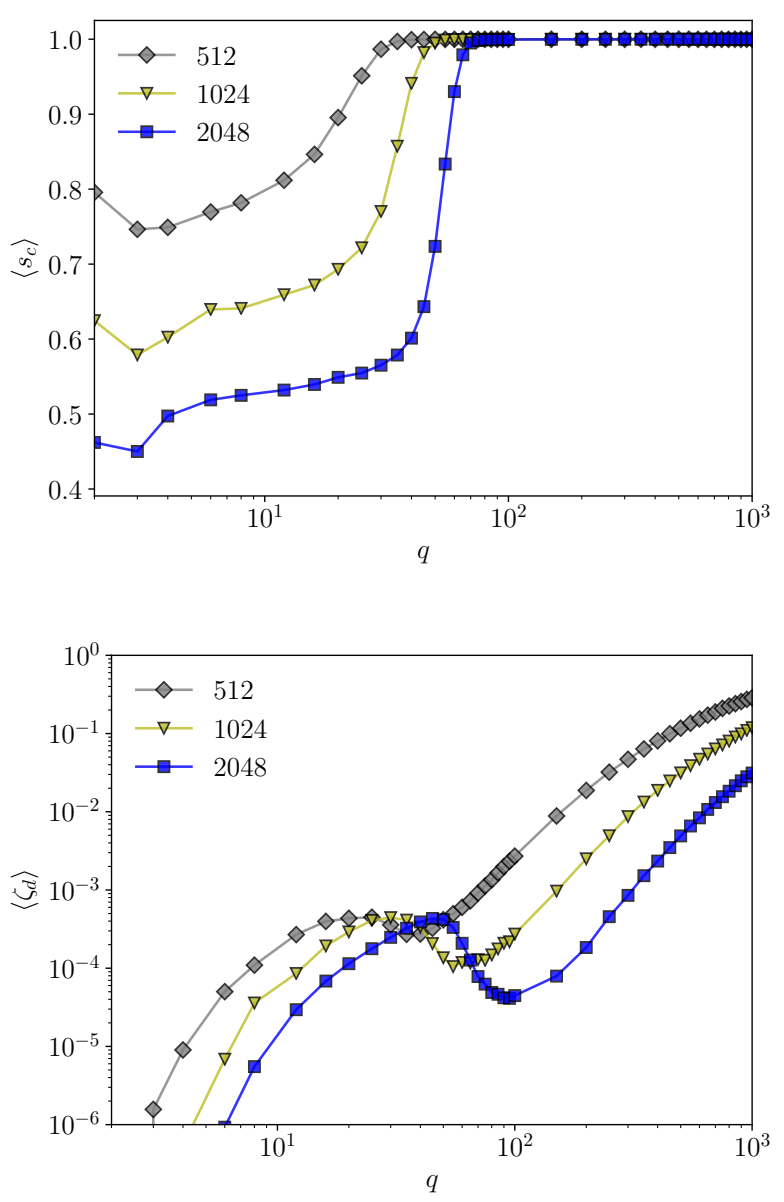

FIG. 15. Mean fraction of agents in the largest component $\left\langle s_{c}\right\rangle$ (upper panel) and mean fraction of culturally isolated agents $\left\langle\zeta_{d}\right\rangle$ (lower panel) as functions of the initial cultural diversity $q$ for $\delta=7$ and systems of size $N=2^{l}$ with $l=$ $9,10,11$, as indicated. of the non-interacting agents is noteworthy for very large $q$ only. More explicitly, since for $\delta>0$ an agent can, in principle, enter the influence neighborhood of any other agent in the square box, the probability that a particular agent becomes culturally isolated in the initial setup of the system is $(1-1 / q)^{N F} \approx \exp (-N F / q)$ so that $q$ must be on the order of $N F$ to guarantee that $\left\langle\zeta_{d}\right\rangle$ is on the order of 1 .

\section{Appendix B: Topology of the absorbing configurations}

The initial topology of the influence network is a random geometric graph (RGG), but the comfort-driven mobility leads to absorbing configurations that are characterized, in principle, by different topologies. Here we offer a brief characterization of those topologies focusing on the mean degree (i.e., the mean number of links an agent has to other agents) of the influence network, which is the simplest and most impacting property of the topology. Figure 16 shows the difference between the mean degree of the absorbing and the initial configurations for $\delta=0.4$ and $\delta=5$ (see Figs. 6 and 8 for the size of the largest component corresponding to these step size values). We recall that for $\alpha=1.75$ and $\rho=1$, the mean degree of the initial RGGs is $\left\langle k_{i}\right\rangle \approx 9.62$.

A first point to be noted in Fig. 16 is that the mean degree tends smoothly to its asymptotic value in the thermodynamic limit, so the fragmentation phase transitions have no consequences on the first moment of the degree distribution. For $\delta=0.4$ (upper panel), we note that the absorbing configurations of the severely fragmented regime exhibit a significantly larger degree than those of the mildly fragmented regime, which have a mean degree very close to that of the RGGs. This result implies a high density of agents within the components and, in fact, there is a high correlation between the mean degree and the density of agents within the largest component (see lower panels of Figs. 6 and 8). It is curious that the mean degree increases with increasing $N$ for $\delta=0.4$, but decreases with $N$ for $\delta=5$. Moreover, the regions with the strongest finite size effects are reversed for these values of $\delta$. For fixed $q$, the mean degree of the influence network increases with increasing $\delta$, which means that one should expect the clusters of agents to be more compact for large $\delta$, in agreement with the spatial organization depicted in the snapshots of the absorbing configurations of Fig. 13.

The fragmentation of the influence network in components implies that the density of agents is not homogeneous along the square box (see Fig. 13). We recall that the density of agents is homogeneous in the initial configurations for all $\delta$ as well as in the absorbing configurations for $\delta=0$, since the influence networks are RGGs in those cases. In Fig. 17 we quantify the spatial distribution of the agents by calculating the fraction of pairs of agents whose distance is between $r$ and $r+\delta r$, which we denote by $g_{\delta}(r) \delta r$. Clearly, $g_{\delta}(r)$ is the usual 

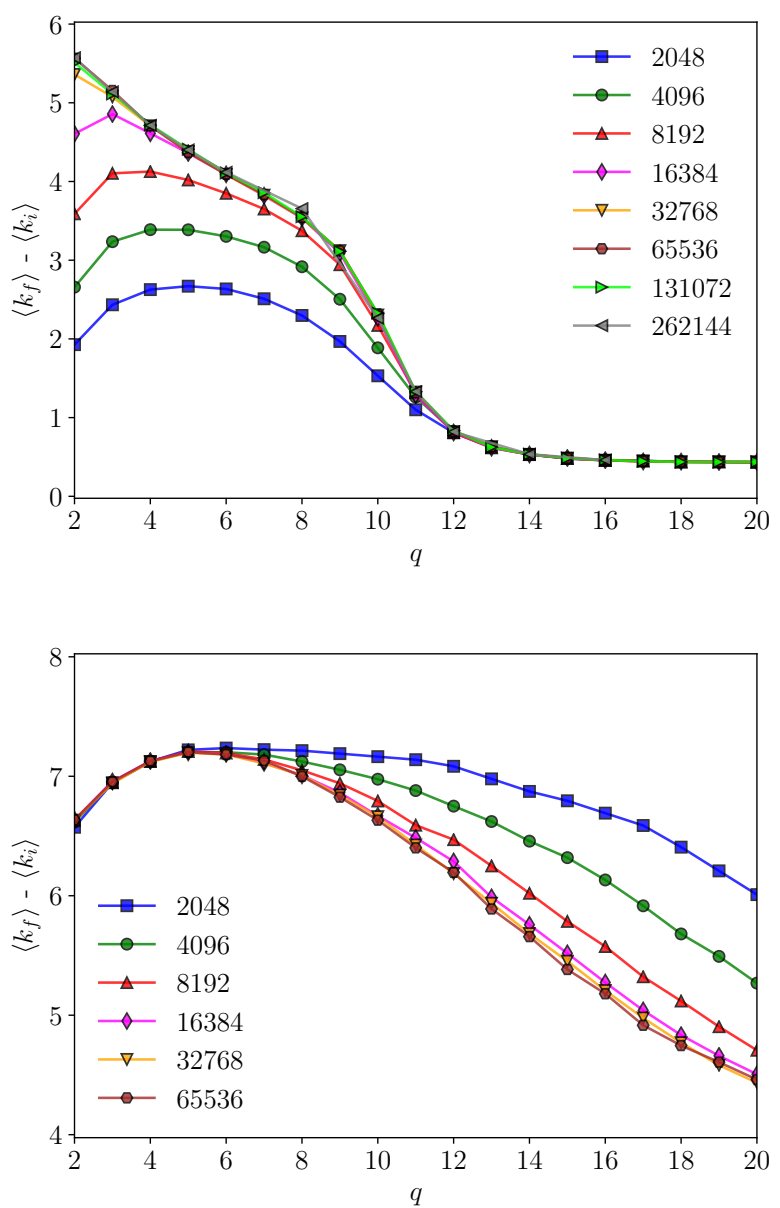

FIG. 16. Difference between the final and initial mean degree of the influence networks as a function of the initial cultural diversity $q$ for $\delta=0.4$ (upper panel) and $\delta=5$ (lower panel) and systems of size $N$ as indicated.

pair correlation function of Statistical Mechanics. In this figure we use $\delta r=0.01$ for a system of $N=2^{16}$ agents, so the length of the sides of the square box is $L=256 \gg \delta r$. In addition, for $q=10$ we pick representative values of the step size, viz. $\delta=0.2,1,8$, that lead to the different fragmentation scenarios revealed in Figs. 11 and 12 . For the RGGs of the static case we have $g_{0}(r)=2 \pi r / N$ for not too large $r$. Most interestingly, $g_{\delta}(r)$ exhibits a discontinuity at $r=\alpha$ for $\delta>0$. Since the absorbing configurations produced by the comfort-driven mobility exhibit a greater number of agents separated by distances $r<\alpha$ compared to the RGGs, this type of mobility results in an effective attraction between culturally similar agents. In the case of $\delta=0.2$, which corresponds to fragmented networks, we observe a high density of pairs of agents separated by distances less than $r=\alpha$ and a low density of pairs separated by distances around $r=2 \alpha$. Of course, the pair densities are high or low depending on whether they are greater or less than the pair density of the RGG. The two absorbing configurations depicted in Fig. 13 illustrate quite nicely the results of Fig. 17 since we can see that the clusters are denser and larger for $\delta=5.6$ than for $\delta=0.2$. Moreover, for $\delta=0.2$ the rarified regions, corresponding to the dip at $r \approx 2 \alpha$ of the normalized pair correlation function, are as conspicuous as the clusters, corresponding to the peak at $r \approx 0.2$ (see lower panel of Fig. 17).
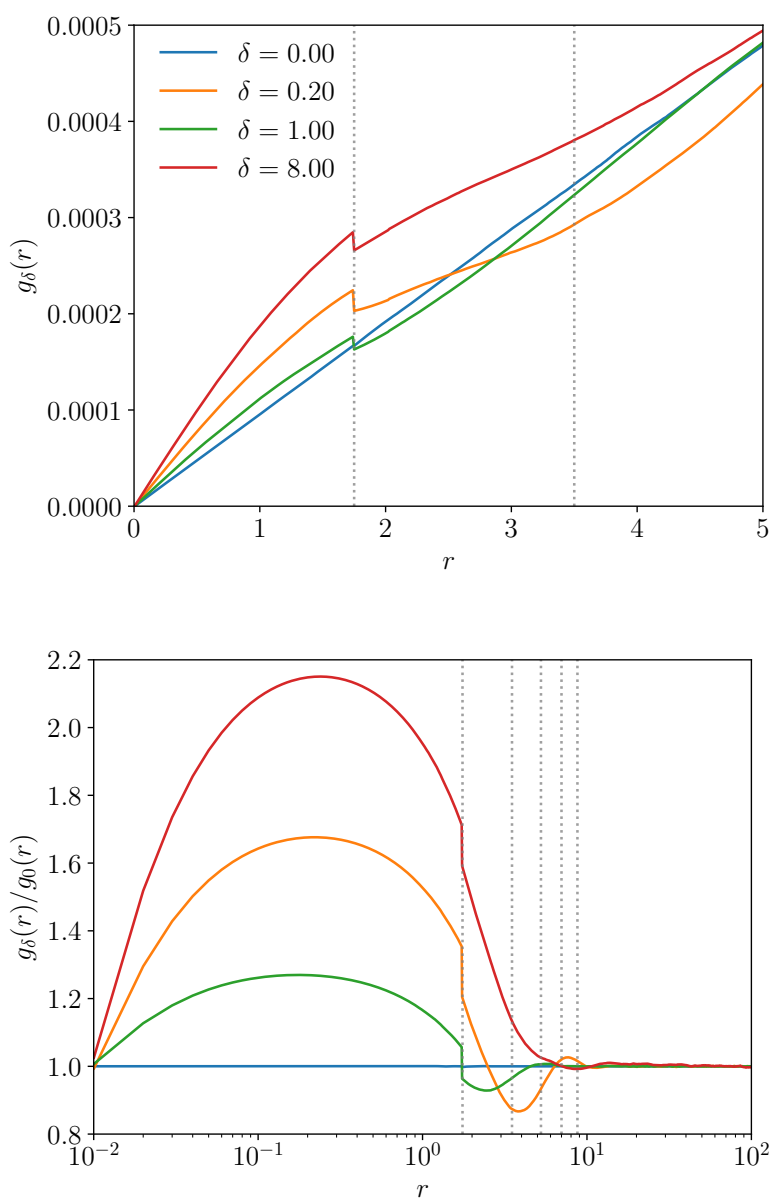

FIG. 17. Pair correlation function $g_{\delta}$ (lower panel)) for the comfort-driven mobility with step sizes $\delta=0,0.2,1,8$, as indicated. The upper panel shows the ratio $g_{\delta} / g_{0}$ where $g_{0}(r) \approx 2 \pi r / N$ is the pair correlation function for the RGGs $(\delta=0)$. The system size is $N=2^{16}$ and the initial cultural diversity is $q=10$. The vertical dotted lines are $r=\alpha, 2 \alpha, \ldots, 5 \alpha$ with $\alpha=1.75$.
[1] Lazarsfeld P, Berelson B and Gaudet H 1948 The People's Choice (New York: Columbia University Press)
[2] Castellano C, Fortunato S and V. Loreto V 2009 Statistical physics of social dynamics Rev. Mod. Phys. $\mathbf{8 1}$ 
591

[3] Galam S 2012 Sociophysics (New York: Springer)

[4] Axelrod R 1997 The Dissemination of Culture: A Model with Local Convergence and Global Polarization J. Conflict Res. 41203

[5] Klemm K, Eguíluz V M, Toral R and San Miguel M Phys. Rev. E 67, 026120 (2003).

[6] Klemm K, Eguíluz V M, Toral R and San Miguel M 2003 Role of dimensionality in Axelrod's model for the dissemination of culture Physica A $\mathbf{3 2 7} 1$

[7] Klemm K, Eguíluz V M, Toral R and San Miguel M 2003 Global culture: A noise-induced transition in finite systems Phys. Rev. E 67 045101(R)

[8] Klemm K, Eguíluz V M, Toral R and San Miguel M 2005 Globalization, polarization and cultural drift J. Econ. Dynam. Control 29321

[9] Reia S M and Fontanari J F 2016 Effect of long-range interactions on the phase transition of Axelrod's model Phys. Rev. E 94052149

[10] Zimmermann M G, Eguíluz V M and San Miguel M 2004 Coevolution of dynamical states and interactions on networks Phys. Rev. E 69 065102(R)

[11] Pacheco J M, Traulsen A and Nowak M A 2006 Coevolution of strategy and structure in complex networks with dynamical linking Phys. Rev. Lett. 97258103

[12] Vazquez F, González-Avella J C, Eguíluz V M and San Miguel M 2007 Time scale competition leading to fragmentation and recombination transitions in the coevolution of network and states Phys. Rev. E 76046120

[13] Vazquez F, Eguíluz V M and San Miguel M 2008 Generic absorbing transition in coevolution dynamics Phys. Rev. Lett. 100108702

[14] Kimura D and Hayakawa Y 2008 Coevolutionary networks with homophily and heterophily Phys. Rev. E $\mathbf{7 8}$ 016103

[15] Herrera J L, Cosenza M G, Tucci K and González-Avella J C 2011 General coevolution of topology and dynamics in networks Europhys. Lett. 9558006

[16] Min B and San Miguel M 2017 Fragmentation transitions in a coevolving nonlinear voter model Sci. Rep. 712864

[17] Murase Y, Jo H-H, Török J, Kertész J and Kaski K 2019 Structural transition in social networks: the role of homophily Sci. Rep. 94310

[18] Benatti A, Arruda H F, Silva F N, Comin C H and Costa L F 2019 Opinion Diversity and Social Bubbles in Adaptive Sznajd Networks arXiv:1905.00867

[19] Gilbert E 1961 Random Plane Networks J. Soc. Indust. Appl. Math. 9533

[20] Penrose M 2003 Random geometric graphs (Oxford: Oxford University Press)
[21] Dall J and Christensen M 2002 Random geometric graphs Phys. Rev. E 66016121

[22] Díaz-Guilera A, Gómez-Gardeñes J, Moreno Y and Nekovee M 2009 Synchronization in random geometric graphs Int. J. Bif. Chaos 19687

[23] Zhang W, Lim C C, Korniss G and Szymanski B K 2014 Opinion dynamics and influencing on random geometric graphs Sci. Rep. 45568

[24] Estrada E, Meloni S, Sheerin M, and Moreno Y 2016 Epidemic spreading in random rectangular networks Phys. Rev. E 94052316

[25] Reia S M, Gomes P F and Fontanari J F 2019 Policies for allocation of information in task-oriented groups: elitism and egalitarianism outperform welfarism Eur. Phys. J. B 92205

[26] Zhao K, Stehlé J, Bianconi G, and Barrat A 2011 Social network dynamics of face-to-face interactions Phys. Rev. E 83056109

[27] Starnini M, Baronchelli A, and Pastor-Satorras R 2013 Modeling Human Dynamics of Face-to-Face Interaction Networks Phys. Rev. Lett. 110168701

[28] Gomes P F, Reia S M , Rodrigues F A and Fontanari J F 2019 Mobility helps problem-solving systems to avoid groupthink Phys. Rev. E 99032301

[29] Castellano C, Marsili M and Vespignani A 2000 Nonequilibrium Phase Transition in a Model for Social Influence Phys. Rev. Lett. 853536

[30] Peres L R and Fontanari J F 2015 The nature of the continuous non-equilibrium phase transition of Axelrod's model Europhys. Lett. 11158001

[31] Vilone D, Vespignani A and Castellano C 2002 Ordering phase transition in the one-dimensional Axelrod model Europ. Phys. J. B 30399

[32] Biral E J P, Tilles P F C and Fontanari J F 2015 The consensus in the two-feature two-state one-dimensional Axelrod model revisited J. Stat. Mech. P04006

[33] Binder K 1981 Critical Properties from Monte Carlo Coarse Graining and Renormalization Phys.Rev. Lett. 47 693

[34] Festinger L, Schachter S and Back K 1963 Social Pressure in Informal Groups: A Study of Human Factors in Housing (Stanford: Stanford University Press)

[35] Reijula S and J. Kuorikoski J 2019 Modeling epistemic communities, in The Routledge Handbook of Social Epistemology, edited by Fricker M, Graham P J, Henderson D, Pedersen N J L L ( Abingdon, UK: Routledge), pp. 277-308

[36] Goldstone R L and Janssen M A 2005 Computational models of collective behavior Trends Cogn. Sci. 9424 